Department of Social Systems and Management

\author{
Discussion Paper Series
}

No. 1254

Development of Computational Algorithms for

Pricing European Bond Options under the Influence of Macro-economic Conditions

\author{
by \\ Jia-Ping Huang and Ushio Sumita
}

February 2010

UNIVERSITY OF TSUKUBA

Tsukuba, Ibaraki 305-8573

JAPAN 


\title{
Development of Computational Algorithms for Pricing European Bond Options under the Influence of Macro-economic Conditions
}

\author{
Jia-Ping HUANG ${ }^{1} \quad$ Ushio SUMITA ${ }^{2}$
}

February 18, 2010

${ }^{1}$ University of Tsukuba; Post Address: Graduate School of Systems and Information Engineering, University of Tsukuba, 1-1-1 Tennoudai, Tsukuba, Ibaraki 305-8573, JAPAN; Email: kou20@sk.tsukuba.ac.jp; Tel: +81 (0)9061775559.

${ }^{2}$ University of Tsukuba; Post Address: Graduate School of Systems and Information Engineering, University of Tsukuba, 1-1-1 Tennoudai, Tsukuba, Ibaraki 305-8573, JAPAN; Email: sumita@sk.tsukuba.ac.jp; Tel: +81 (0)29 8535096. 


\begin{abstract}
A stochastic process of Vasicek type describing the short rate is considered, where the three governing parameters $\{\phi, \alpha, \sigma\}$, with $\phi$ for the market fitting, $\alpha$ for the reversion and $\sigma$ for the volatility, would depend on the macro-economic condition modeled as an independent birth-death process on a finite state space. Computational algorithms are developed for evaluating the prices of European call options defined on a zero-coupon discount bond characterized by the above stochastic process. Numerical examples are provided based on real data so as to demonstrate the speed and efficiency of the proposed algorithms.
\end{abstract}

Keywords: Short rate process, Vasicek model, Macro-economic condition, Ehrenfest approximation, Uniformization procedure, Computational Algorithms for Pricing, European call option. 


\section{Introduction}

One of the most prevalent one factor term structure models for pricing interest rate options is the Hull-White model characterized by the stochastic differential equation

$$
d R(t)=\{\phi(t)-\alpha(t) R(t)\} d t+\sigma(t) d W(t)
$$

where $R(t)$ is a random short rate, $W(t)$ is the standard Wiener process, $\phi(t)$ is the market fitting function, $\alpha(t)$ is the reversion function and $\sigma(t)$ is the volatility function. When both $\alpha(t)$ and $\sigma(t)$ are constant, Hull-White (1990a) show the explicit formulas for evaluating the prices of zero-coupon discount bonds and associated European options. When $\alpha(t)$ and $\sigma(t)$ are time-dependent, however, such formulas are not available in a closed form and the prices cannot be obtained easily.

Jin, Gotoh and Sumita (2007) develop computational algorithms for pricing European options associated with $R(t)$ in (1.1) where $\alpha(t)$ and $\sigma(t)$ are approximated by step functions. Their approach is based on the Ehrenfest approximation, established in Sumita, Gotoh and Jin (2006), of the Ornstein-Uhlebeck (O-U) process $\left\{\widehat{X}_{O U}(t): t \geq 0\right\}$ which constitutes the classic Vasicek model, see Vasicek (1977), characterized by

$$
d \widehat{X}_{O U}(t)=\left\{\phi-\alpha \widehat{X}_{O U}(t)\right\} d t+\sigma d W(t)
$$

Using the uniformization procedure of Keilson (1979), efficient computational algorithms are first established in Sumita, Gotoh and Jin (2006) for pricing European options associated with $\widehat{X}_{O U}(t)$. Then, in Jin, Gotoh and Sumita (2007), a single process $R(t)$ with time varying parameters $\alpha(t)$ and $\sigma(t)$ is replaced by a sequence of distinct processes $\widehat{X}_{O U}(t)$ 's, each defined in a time interval in which both $\alpha(t)$ and $\sigma(t)$ are constant. Such segmented $\widehat{X}_{O U}(t)$ 's are connected together at the end points of the time intervals. The Vasicek model computations are patched together accordingly through a sequence of the Ehrenfest approximations. Based on the same Ehrenfest approximation, Gotoh, Jin and Sumita (2009) propose computational procedures for pricing barrier options associated with $\widehat{X}_{O U}(t)$ in $(1.2)$.

For practical purposes, it is natural to consider a case in which an external stochastic process describing the macro-economic condition would affect the underlying parameter functions of the Hull-White model. More specifically, let $\{J(t): t \geq 0\}$ be a Markov chain in continuous time on $\mathcal{J}=\{0, \cdots, J\}$ governed by hazard matrix $\underline{\eta}=\left[\eta_{i j}\right]$. We consider a stochastic differential equation specified by

$$
d \widehat{X}_{O U: J(t)}(t)=\left\{\phi_{J(t)}-\alpha_{J(t)} \widehat{X}_{O U: J(t)}(t)\right\} d t+\sigma_{J(t)} d W(t) .
$$

To the best knowledge of the authors, this stochastic process has not been analyzed in the literature. The purpose of this paper is to develop computational algorithms for pricing European options associated with the random short rate $\widehat{X}_{O U: J(t)}(t)$ based on the Ehrenfest approximation of Sumita, Gotoh and Jin (2006).

The structure of this paper is as follows. In Section 2, a succinct summary of the Ehrenfest approximation is provided from Sumita, Gotoh and Jin (2006). Computational procedures are then developed in Section 3 for evaluating the time-dependent joint probability of $\left[J(t), \widehat{X}_{O U: J(t)}(t)\right]$ through the dynamic stochastic mixture of the underlying 
Ehrenfest approximations. It is shown in Section 4 that European call option associated with the random short rate $\widehat{X}_{O U: J(t)}(t)$ can be evaluated based on the results of Section 3. Numerical examples are given in Section 5. Firstly, based on LIBOR (London InterBank Offered Rate), the upward hazard rate and the downward hazard rate of $J(t)$ on $\mathcal{J}=\{-1,0,1\}$ are estimated. Next, using LIBOR, the Vasicek model parameters $\phi_{j}, \alpha_{j}$ and $\sigma_{j}$ are estimated for each $j \in \mathcal{J}$. Prices for a class of European call options as-

sociated with $\widehat{X}_{O U: J(t)}(t)$ are then computed, demonstrating speed and accuracy of the computational algorithms developed in this paper.

\section{Ehrenfest Approximation of O-U Process}

In this section, we provide a succinct summary of the Ehrenfest approximation of the O-U process $\widehat{X}_{O U}(t)$ characterized by (1.2) based on Sumita, Gotoh and Jin (2006). We first consider the stochastic process $X_{O U}(t)$ obtained from (1.2) by setting $\phi=0$, i.e.

$$
d X_{O U}(t)=-\alpha X_{O U}(t) d t+\sigma d W(t) .
$$

The relationship between $\widehat{X}_{O U}(t)$ in $(1.2)$ and $X_{O U}(t)$ in (2.1) can be found, after a little algebra, as

$$
\widehat{X}_{O U}(t)=X_{O U}(t)+\theta(t)
$$

where $X_{O U}(0)=0$ and

$$
\theta(t) \stackrel{\text { def }}{=} \frac{\phi}{\alpha}\left(1-\mathrm{e}^{-\alpha t}\right)+\widehat{X}_{O U}(0) \mathrm{e}^{-\alpha t}
$$

Since $\theta(t)$ is a one-to-one mapping, the transition probabilities of $\widehat{X}_{O U}(t)$ can be constructed from those of $X_{O U}(t)$. It is shown in Sumita, Gotoh and Jin (2006) that a certain sequence of Ehrenfest processes converges in law to $X_{O U}(t)$ as the corresponding discretized state space becomes dense in the limit. Using the uniformization procedure of Keilson (1979), the transition probabilities of the Ehrenfest process approximating $X_{O U}(t)$ can be computed with speed and accuracy, which in turn enables one to evaluate the transition probabilities of $\widehat{X}_{O U}(t)$ based on (2.2) and (2.3). Prices of European call options associated with $\widehat{X}_{O U}(t)$ can then be obtained by employing the above computational procedures.

More specifically, let $X_{O U}(t)$ be the stochastic process characterized by (2.1). We approximate $X_{O U}(t)$ by considering a sequence of Ehrenfest processes $\left\{N_{V}(t): t \geq 0\right\}$ defined on $\mathcal{N}_{V}=\{0,1, \cdots, 2 V\}$ governed by upward transition rates $\lambda_{n}$ and downward transition rates $\mu_{n}$ given by

$$
\lambda_{n}=\frac{\alpha}{2}(2 V-n), \quad \mu_{n}=\frac{\alpha n}{2}, \quad n \in \mathcal{N}_{V} .
$$

It should be noted that

$$
\nu_{n}=\lambda_{n}+\mu_{n}=\alpha V, \quad n \in \mathcal{N}_{V},
$$

which is independent of $n$. 
In order to position the state space $\mathcal{N}_{V}$ of $N_{V}(t)$ in the state space $\{-\infty, \infty\}$ of $X_{O U}(t)$, we introduce a sequence of intermediary stochastic processes $\left\{X_{V}(t): t \geq 0\right\}, V=$ $1,2,3, \cdots$, defined by

$$
X_{V}(t) \stackrel{\text { def }}{=} \frac{\sigma}{\sqrt{\alpha V}} N_{V}(t)-\sigma \sqrt{\frac{V}{\alpha}} .
$$

It should be noted that the state space $\mathcal{X}_{V}$ of $X_{V}(t)$ can be written as $\mathcal{X}_{V}=\left\{x_{V}(0), \cdots, x_{V}(2 V)\right\}$ where

$$
x_{V}(n)=\frac{\sigma}{\sqrt{\alpha V}} n-\sigma \sqrt{\frac{V}{\alpha}}, \quad n \in \mathcal{N}_{V} .
$$

The correspondence between the states of $N_{V}(t)$ and those of $X_{V}(t)$ is summarized in Table 1, where

$$
\xi_{V}(x)=\left\lceil\frac{\sqrt{\alpha V}}{\sigma} x\right\rceil,
$$

with $\lceil a\rceil$ denoting the smallest integer which is greater than or equal to $a$.

[Table 1 about here.]

It is shown in Sumita, Gotoh and Jin (2006) that $X_{V}(t)$ converges in law to $X_{O U}(t)$ as $V \rightarrow \infty$. Furthermore, the first passage time and the historical maximum of $X_{V}(t)$ also converge in law to those of $X_{O U}(t)$ as $V \rightarrow \infty$. These results provide a computational vehicle for this paper, as we will see.

\section{Development of Short Rate Model under the In- fluence of Macro-economic Conditions}

We consider a stochastic process $\{J(t): t \geq 0\}$ describing the marco-economic condition at time $t$, which would affect the random short rate and therefore prices of the associated derivatives. Throughout the paper, it is assumed that $J(t)$ is a continuous time birthdeath process on $\mathcal{J}=\{0, \cdots, J\}$ governed by hazard rate matrix $\underline{\underline{\eta}}=\left[\eta_{i j}\right]$, where $\underline{\underline{\eta}}$ is given by

$$
\underline{\underline{\eta}}=\left[\begin{array}{ccccccc}
0 & \eta_{0}^{+} & & & & & \\
\eta_{1}^{-} & 0 & \eta_{1}^{+} & & & \underline{0} & \\
& & \ddots & & & & \\
& & \eta_{j}^{-} & 0 & \eta_{j}^{+} & & \\
& & & & \ddots & & \\
& \underline{0} & & & \eta_{J-1}^{-} & 0 & \eta_{J-1}^{+} \\
& & & & & \eta_{J}^{-} & 0
\end{array}\right] .
$$

Of interest is the random short rate $\widehat{X}_{O U: J(t)}(t)$ characterized by

$$
d \widehat{X}_{O U: J(t)}(t)=\left\{\phi_{J(t)}-\alpha_{J(t)} \widehat{X}_{O U: J(t)}(t)\right\} d t+\sigma_{J(t)} d W(t)
$$


It should be noted that, given $J(t)=j$, the process $\widehat{X}_{O U: J(t)}(t)$ behaves like a Vasicek model with constant parameters $\phi_{j}, \alpha_{j}$ and $\sigma_{j}$ until $J(t)$ changes its state. Accordingly, $\widehat{X}_{O U: J(t)}(t)$ can be interpreted as a dynamic random mixture of Vasicek models. The purpose of this section is to prepare a basis for developing computational procedures to evaluate the time-dependent joint probability of $\left[J(t), \widehat{X}_{O U: J(t)}(t)\right]$, which would be discussed in the next section.

To understand the stochastic characteristics of $\widehat{X}_{O U: J(t)}(t)$, we need to introduce the age process $Y(t)$ associated with the underlying Markov process $J(t)$, that is, with $\tau_{n}$ denoting the $n$-th transition epoch of $J(t)$ for $n \geq 0$ with $\tau_{0}=0$, one has

$$
Y(t)=t-\max \left\{\tau_{n}: 0 \leq \tau_{n} \leq t\right\}
$$

Given $J(t)=j, \widehat{X}_{O U: J(t)}(t)$ behaves like a Vasicek model with constant parameters $\phi_{j}, \alpha_{j}$ and $\sigma_{j}$ until $J(t)$ changes its state. Because $Y(t)$ describes the elapsed time at time $t$ since the last transition of $J(t)$ into the current state, this last transition time can be written as $t-Y(t)$. One then sees, from (2.1) and (2.2), that

$$
\widehat{X}_{O U: j}(t)=X_{O U: j}(Y(t))+\theta_{j}(Y(t)), \quad j \in \mathcal{J},
$$

where

$$
d X_{O U: j}(t)=-\alpha_{j} X_{O U: j}(t) d t+\sigma_{j} d W(t), \quad X_{O U: j}(0)=0
$$

and

$$
\theta_{j}(Y(t)) \stackrel{\text { def }}{=} \frac{\phi_{j}}{\alpha_{j}}\left(1-\mathrm{e}^{-\alpha_{j} Y(t)}\right)+\widehat{X}_{O U: j}(t-Y(t)) \mathrm{e}^{-\alpha_{j} Y(t)}
$$

As in Sumita, Gotoh and Jin (2006), the joint probability of $\left[J(t), \widehat{X}_{O U: J(t)}(t)\right]$ can be derived from that of $\left[J(t), Y(t), X_{O U: J(t)}(Y(t))\right]$ from (3.4), provided that the values of $\widehat{X}_{O U: J\left(\tau_{n}\right)}\left(\tau_{n}\right), 0 \leq \tau_{n} \leq t, n \geq 0$, are known.

Following the discussion of Section 2, the process $X_{O U: j}(y)$ with $j \in \mathcal{J}$ can be approximated by the Ehrenfest process $\left\{N_{V: j}(y): y \geq 0\right\}$ on $\mathcal{N}_{V}=\{0,1, \cdots, 2 V\}$ governed by upward transition rates $\lambda_{j: n}$ and downward transition rates $\mu_{j: n}$ where

$$
\lambda_{j: n}=\frac{\alpha_{j}}{2}(2 V-n), \quad \mu_{j: n}=\frac{\alpha_{j} n}{2}, \quad n \in \mathcal{N}_{V} .
$$

The corresponding intermediary stochastic processes $\left\{X_{V: j}(y): y \geq 0\right\}$ are then defined as

$$
X_{V: j}(y) \stackrel{\text { def }}{=} \frac{\sigma_{j}}{\sqrt{\alpha_{j} V}} N_{V: j}(y)-\sigma_{j} \sqrt{\frac{V}{\alpha_{j}}},
$$

which allows one to bridge states between $N_{V: j}(y)$ and $X_{O U: j}(y)$, i.e., given $N_{V: j}(y)=n$, one has

$$
x_{V: j}(n)=\frac{\sigma_{j}}{\sqrt{\alpha_{j} V}} n-\sigma_{j} \sqrt{\frac{V}{\alpha_{j}}},
$$


and

$$
\widehat{x}_{V: j}(y, n, t)=x_{V ; j}(n)+\theta_{j}(y, t)
$$

where

$$
\theta_{j}(y, t)=\frac{\phi_{j}}{\alpha_{j}}\left(1-\mathrm{e}^{-\alpha_{j} y}\right)+\widehat{x}_{V: j}(0, V, t-y) \mathrm{e}^{-\alpha_{j} y} .
$$

From the discussion above, it can be seen that the joint probability of $[J(t), Y(t)$, $\left.X_{O U: J(t)}(Y(t))\right]$ can be assessed from that of $\left[J(t), Y(t), N_{V: J(t)}(Y(t))\right]$, which in turn enables one to evaluate $\left[J(t), \widehat{X}_{O U: J(t)}(t)\right]$ of our principal interest. Accordingly, in what follows, we focus on the joint process $\left[J(t), Y(t), N_{V: J(t)}(Y(t))\right]$ for developing algorithmic procedures to compute the joint probability.

\section{Computational Procedures for Evaluating Time- dependent Joint Probability of $\left[J(t), \widehat{X}_{O U: J(t)}(t)\right]$ and Prices of Associated European Bond Options}

We are now in a position to establish computational procedures for evaluating the timedependent joint probability of $\left[J(t), Y(t), N_{V: J(t)}(Y(t))\right]$ based on the Ehrenfest approximation described in Section 2 and the uniformization procedure of Keilson (1979). One can see that, the state transition of the joint process triggered by the state transition of $J(t)$ is affected by the age $Y(t)$ of $J(t)$ prior to the transition. Because of this, it is necessary to consider the embedded process $\left[J^{\#}(k), Y^{\#}(k), N_{V: J^{\#}(k)}^{\#}(Y(k))\right]$ with $t=k \Delta$, $k=0,1,2, \cdots$ for some $\Delta>0$, where it is assumed that $\Delta$ is small enough so that no transition of $J(t)$ would occur in $[k \Delta,(k+1) \Delta)$ and $J(t)$ could change its state only at discrete points $k \Delta$. Since $J(t)$ describes the macro-economic condition, this assumption is not so unreasonable, e.g., for many applications, it would be sufficient to consider the macro-economic condition that may change at least in time unit of a month. $J^{\#}(k)$ can be constructed from $J(t)$ via the uniformization procedure of Keilson (1979). More specifically, the one step transition probability matrix $\underline{\underline{P}}_{J \#}$ of $J^{\#}(k)$ can be expressed in terms of the transition probability matrix $\underline{\underline{P}}_{J}(t)$ of $J(t)$ as

$$
\underline{\underline{P}}_{J \#}=\left[p_{J \#:(i, j)}\right]_{i, j \in \mathcal{J}}=\underline{\underline{P}}_{J}(\Delta) .
$$

For evaluating $\underline{\underline{P}}_{J \#}$, let $\underline{\underline{\eta}}_{D}$ be defined by

$$
\underline{\underline{\eta}}_{D}=\operatorname{diag}\left[\eta_{j}\right]_{j \in \mathcal{J}}, \quad \eta_{j}= \begin{cases}\eta_{0}^{+} & j=0 \\ \eta_{j}^{-}+\eta_{j}^{+} & 1 \leq j \leq J-1, \\ \eta_{j}^{-} & j=J\end{cases}
$$

where $\eta_{j}^{+}, \eta_{j}^{-}$are given in (3.1). Let $\eta \geq \max _{j \in \mathcal{J}}\left\{\eta_{j}\right\}$ and let $\underline{a}_{\eta}$ be a stochastic matrix specified by

$$
\underline{\underline{a}}_{\eta}=\underline{\underline{I}}-\frac{1}{\eta} \underline{\underline{\eta}} D+\frac{1}{\eta} \underline{\underline{\eta}}
$$


Following the uniformization procedure of Keilson (1979), the transition probability matrix of $J(t)$ can be written as

$$
\underline{\underline{P}}_{J}(t)=\exp \left\{-\eta t\left[\underline{\underline{I}}-\underline{\underline{a}}_{\eta}\right]\right\}=\sum_{r=0}^{\infty} \mathrm{e}^{-\eta t} \frac{(\eta t)^{r}}{r !} \underline{\underline{a}}_{\eta}^{r},
$$

where $\underline{\underline{A}}^{0}=\underline{\underline{I}}$ for any square matrix $\underline{\underline{A}}$. From (4.1), one has

$$
\underline{\underline{P}}_{J \#}=\sum_{r=0}^{\infty} \mathrm{e}^{-\eta \Delta} \frac{(\eta \Delta)^{r}}{r !} \underline{\underline{a}}_{\eta}^{r} \text {. }
$$

In $[k \Delta,(k+1) \Delta)$ and given that $J^{\#}(k)=j$ and $Y^{\#}(k)=\ell$, the marginal process $N_{V: j}(\ell)$ behaves like an ordinary Markov chain in continuous time. Accordingly, the one step transition probability matrix $\underline{\underline{P}}_{N_{V: j}^{\#}}$ of the embedded process $N_{V: j}^{\#}(k)$ can be expressed in terms of the transition probability matrix $\underline{\underline{P}}_{N_{V: j}}(t)$ of $N_{V: j}(t)$ as

$$
\underline{\underline{P}}_{N_{V: j}^{\#}}^{\#}=\left[p_{N_{V: i}^{\#}:(m, n)}\right]_{m, n \in \mathcal{N}_{V}}=\underline{\underline{P}}_{N_{V: j}}(\Delta) \text {. }
$$

Evaluation of $\underline{\underline{P}}_{N_{V: j}^{\#}}$ is similar to that of $\underline{\underline{P}}_{J \#}$. More specifically, let $\underline{\nu}_{j}$ be the hazard rate matrix of $N_{V: j}(t)$ given by

$$
\underline{\nu}_{j}=\left[\begin{array}{ccccccc}
0 & \lambda_{j: 0} & & & & \\
\mu_{j: 1} & 0 & \lambda_{j: 1} & & & \underline{0} & \\
& & \ddots & & & & \\
& & \mu_{j: n} & 0 & \lambda_{j: n} & & \\
& & & \ddots & & \\
& \underline{0} & & & \mu_{j: 2 V-1} & 0 & \lambda_{j: 2 V-1} \\
& & & & & \mu_{j: 2 V} & 0
\end{array}\right],
$$

where $\lambda_{j: n}$ and $\mu_{j: n}$ are defined in (3.7). For $\underline{\underline{\nu}}_{D: j}$ given by

$$
\underline{\underline{\nu}}_{D: j}=\operatorname{diag}\left[\nu_{j: n}\right]_{n \in \mathcal{N}_{V}}, \quad \nu_{j: n}= \begin{cases}\lambda_{j: 0} & n=0 \\ \mu_{j: n}+\lambda_{j: n} & 1 \leq n \leq 2 V-1 \\ \mu_{j: 2 V} & n=2 V\end{cases}
$$

let $\nu_{j} \geq \max _{n \in \mathcal{N}_{V}}\left\{\nu_{j: n}\right\}$ and define

$$
\underline{\underline{a}}_{\nu_{j}}=\underline{\underline{I}}-\frac{1}{\nu_{j}} \underline{\nu}_{D: j}+\frac{1}{\nu_{j}} \underline{\nu}_{j} .
$$

Then the transition probability matrix of $N_{V: j}(t)$ can be written as

$$
\underline{\underline{P}}_{N_{V: j}}(t)=\exp \left\{-\nu_{j} t\left[\underline{\underline{I}}-\underline{\underline{a}}_{\nu_{j}}\right]\right\}=\sum_{r=0}^{\infty} \mathrm{e}^{-\nu_{j} t} \frac{\left(\nu_{j} t\right)^{r}}{r !} \underline{\underline{a}}_{\nu_{j}}^{r} .
$$

It then follows from (4.5) that

$$
\underline{\underline{P}}_{N_{V: j}^{\#}}=\sum_{r=0}^{\infty} \mathrm{e}^{-\nu_{j} \Delta} \frac{\left(\nu_{j} \Delta\right)^{r}}{r !} \underline{a}_{\nu_{j}}^{r}
$$


Since $\underline{\underline{a}}_{\eta}$ and $\underline{\underline{a}}_{\nu_{j}}$ are stochastic matrices, the series representation of (4.4) and (4.9) involve only additions and multiplications of non-negative numbers, providing computational stability with speed and accuracy.

Let $\pi(j, \ell, n, k)$ be the probability that $J^{\#}(k)=j, Y^{\#}(k)=\ell$ and $N_{V: J^{\#}}^{\#}\left(Y^{\#}(k)\right)=n$ at time $k \Delta$, i.e.,

$$
\pi(j, \ell, n, k)=\mathrm{P}\left[J^{\#}(k)=j, Y^{\#}(k)=\ell, N_{V: j}^{\#}(\ell)=n\right] .
$$

For $\ell>0$, one sees that

$$
\pi(j, \ell, n, k)=\underline{\underline{P}}_{J \#}(j, j) \sum_{m \in \mathcal{N}_{V}} \pi(j, \ell-1, m, k-1) \underline{\underline{P}}_{N_{V: j}^{\#}}(m, n) .
$$

The above equation can be interpreted probabilistically in the following manner. The trivariate process $\left[J^{\#}(k), Y^{\#}(k), N_{V: J \#(k)}^{\#}\left(Y^{\#}(k)\right)\right]$ is at state $[j, \ell-1, m]$ at time $(k-1) \Delta$ with probability $\pi(j, \ell-1, m, k-1)$. A self-transition of $J^{\#}$ describing the macro-economic condition occurs with probability $\underline{\underline{P}}_{J \#}(j, j)$. Consequently, the trivariate process moves from state $[j, \ell-1, m]$ to state $[j, \ell, n]$ at time $k \Delta$ with probability $\underline{\underline{P}}_{J \#}(j, j) \underline{\underline{P}}_{N_{V: j}^{\#}}(m, n)$. Equation (4.11) then follows by summing over $m \in \mathcal{N}_{V}$.

For $\ell=0$, in parallel with (4.11), one has

$$
\pi(j, 0, n, k)=\delta_{n V} \sum_{i \in \mathcal{J} \backslash\{j\}} \sum_{\ell=0}^{k-1} \sum_{m \in \mathcal{N}_{V}} \pi(i, \ell, m, k-1) \underline{\underline{P}}_{J \#}(i, j) .
$$

In order to interpret this equation probabilistically, we suppose that the joint process is at state $[i, \ell, m]$ at time $(k-1) \Delta$, which occurs with probability $\pi(i, \ell, m, k-1)$. When $J^{\#}$ moves from $i$ to $j$ with probability $\underline{\underline{P}}_{J \#}(i, j)$, the age is reset to be 0 at time $k \Delta$ if and only if $i \neq j$. By summing the resulting probability over $m \in \mathcal{N}_{V}, \ell \in\{0, \cdots, k-1\}$ and $i \in \mathcal{J} \backslash\{j\}$ with the age reset at $\ell=0$, the probability of the trivariate process reaching state $[j, 0, n]$ at time $k \Delta$ is obtained.

Let $\widehat{\chi}(j, \ell, n, k)$ denote the value of the short rate $\widehat{X}_{O U: J(t)}(k \Delta)$ associated with state $[j, \ell, n]$. For $\ell>0$, one sees, from (3.10) and (3.11), that

$$
\begin{aligned}
\widehat{\chi}(j, \ell, n, k)= & x_{V ; j}(n)+\theta_{j}(\ell \Delta, k \Delta) \\
= & \frac{\sigma_{j}}{\sqrt{\alpha_{j} V}} n-\sigma_{j} \sqrt{\frac{V}{\alpha_{j}}}+ \\
& +\frac{\phi_{j}}{\alpha_{j}}\left(1-\mathrm{e}^{-\alpha_{j} \ell \Delta}\right)+\widehat{\chi}(j, 0, V, k-\ell) \mathrm{e}^{-\alpha_{j} \ell \Delta} .
\end{aligned}
$$

For $\ell=0$, one has

$$
\widehat{\chi}(j, 0, n, k)=\delta_{n V} \sum_{i \in \mathcal{J}} \sum_{\ell=1}^{k} \sum_{m \in \mathcal{N}_{V}} \widehat{\chi}(i, \ell, m, k) \pi(i, \ell, m, k),
$$

The probabilistic interpretation of this equation is somewhat subtle. The instant before the macro-economic condition changes its state, the trivariate process $\left[J^{\#}(k), Y^{\#}(k)\right.$, 
$\left.N_{V: J \#(k)}^{\#}\left(Y^{\#}(k)\right)\right]$ should have been at $[i, \ell, m]$ at time $k \Delta-$ with probability $\pi(i, \ell, m, k)$. The value of the short rate corresponding to that state is then given by $\widehat{\chi}(i, \ell, m, k)$. Hence, the original short rate process can be approximated by setting the initial value of the trivariate process reset with $\ell=0$ at state $[j, 0, n]$ for any $j \in \mathcal{J}$ as the weighted average of $\widehat{\chi}(i, \ell, m, k)$ as shown in (4.14).

The whole computational procedure discussed above is summarized in Algorithm A.2. Once $\pi(j, \ell, n, k)$ and $\widehat{\chi}(j, \ell, n, k)$ are calculated, one can use those to construct a computational vehicle for evaluating the prices of European bond options associated with $\widehat{X}_{O U: J(t)}(t)$.

In order to calculate the prices of the associated European bond options, one needs to evaluate the prices of the zero-coupon discount bond. Here we employ a discrete time backward recursive formula which is similar to that in Jin, Gotoh and Sumita (2007). Let $B(j, \ell, n, k \mid K)$ be the price of the zero-coupon discount bond at time $k \Delta$ with maturity $K \Delta$, where the state of the corresponding discrete time process is at

$$
\left[J^{\#}(k), Y^{\#}(k), N_{V: J \#(k)}^{\#}(Y(k))\right]=[j, \ell, n] .
$$

For $k=K$, one sees that

$$
B(j, \ell, n, K \mid K)=b \quad \text { for all } \quad j \in \mathcal{J}, \ell \leq K, n \in \mathcal{N}_{V},
$$

where $b$ is the face value of the bond at maturity. This face value is usually set to be 1 , which we assume throughout the rest of the paper. For $k<K$, one has

$$
\begin{aligned}
B(j, \ell, n, k \mid K) & =\mathrm{e}^{-\widehat{\chi}(j, \ell, n, k) \Delta}\left\{\sum_{i \in \mathcal{J}} \underline{\underline{P}}_{J \#}(j, i) B(i, 0, V, k+1 \mid K)\right. \\
& \left.+\sum_{m \in \mathcal{N}_{V}} \underline{\underline{P}}_{N_{V: j}^{\#}}(n, m) \underline{\underline{P}}_{J \#}(j, j) B(j, \ell+1, m, k+1 \mid K)\right\} .
\end{aligned}
$$

This algorithm is summarized in Algorithm A.3.

We next evaluate the prices of European options defined on the zero-coupon discount bond discussed above. For discrete economies, it is well known that the price of any security with known payoffs can be viewed as a portfolio of Arrow-Debreu securities and can be priced as the payoff-weighted sum over all states of the prices of the Arrow-Debreu securities, see e.g., Pelsser (2000). Accordingly, we first evaluate the price of an ArrowDebreu security. Let $Q(j, \ell, n, k)$ be the present value at time 0 of the Arrow-Debreu security with maturity price 1 at time $k \Delta$ given state $[j, \ell, n]$. For $k=0$, one has

$$
Q(j, 0, n, 0)=\delta_{n V} \quad \text { for all } \quad j \in \mathcal{J}, n \in \mathcal{N}_{V}
$$

If $k \geq 1$, the value depends on whether or not $\ell=0$. More specifically, for $\ell=0$, one has

$$
\begin{aligned}
& Q(j, 0, n, k) \\
& =\delta_{n V} \sum_{i \in \mathcal{J} \backslash\{j\}} \sum_{\ell=0}^{k-1} \sum_{m \in \mathcal{N}_{V}} \mathrm{e}^{-\widehat{\chi}(i, \ell, m, k-1) \Delta} Q(i, \ell, m, k-1) \underline{\underline{P}}_{J \#}(i, j),
\end{aligned}
$$


and one sees, for $\ell \neq 0$, that

$$
\begin{aligned}
& Q(j, \ell, n, k) \\
& =\sum_{m \in \mathcal{N}_{V}} \mathrm{e}^{-\widehat{\chi}(j, \ell-1, m, k-1) \Delta} Q(j, \ell-1, m, k-1) \underline{\underline{P}}_{N_{V: j}^{\#}}(m, n) \underline{\underline{P}}_{J \#}(j, j) .
\end{aligned}
$$

This procedure for evaluating the price of the Arrow-Debreu security is summarized in Algorithm A.4.

Let $\Pi_{C}(M, S \mid K)$ be the present value at time 0 of the European call option with maturity at time $M \Delta$ and strike price $S$ defined on the zero-coupon discount bond with maturity at time $K \Delta$. The counterpart for that of the European put option is denoted by $\Pi_{P}(M, S \mid K)$. By using Equations (4.15) through (4.19), one has

$$
\begin{aligned}
& \Pi_{C}(M, S \mid K)=\sum_{j \in \mathcal{J}} \sum_{\ell=0}^{M} \sum_{n \in \mathcal{N}_{V}}[B(j, \ell, n, M \mid K)-S]^{+} Q(j, \ell, n, M), \\
& \Pi_{P}(M, S \mid K)=\sum_{j \in \mathcal{J}} \sum_{\ell=0}^{M} \sum_{n \in \mathcal{N}_{V}}[S-B(j, \ell, n, M \mid K)]^{+} Q(j, \ell, n, M),
\end{aligned}
$$

where $[a]^{+}=\max \{a, 0\}$. It should be noted that the following relationship exists between $\Pi_{C}(M, S \mid K)$ and $\Pi_{P}(M, S \mid K)$, which is called the "put-call parity":

$$
\begin{aligned}
\Pi_{C}(M, S \mid K)= & \Pi_{P}(M, S \mid K)+B\left(J^{\#}(0), 0, V, 0 \mid K\right) \\
& -S \cdot B\left(J^{\#}(0), 0, V, 0 \mid M\right) .
\end{aligned}
$$

Hence, it is sufficient to establish the computational procedure for $\Pi_{C}(M, S \mid K)$ only, which we summarize in Algorithm A.5.

\section{Estimation of the Underlying Parameter Values and Numerical Results}

In this section, numerical examples are provided to demonstrate the speed and efficiency of Algorithms A.2 through A.5 given in Section 4. For this purpose, the monthly LIBOR (London Inter-Bank Offered Rate) in US dollars for the period September 1989 - December 2008 would be employed as the fundamental data set so as to specify the birth-death process $J(t)$ for describing the macro-economic condition, as well as the Vasicek model $\widehat{X}_{O U: J(t)}(t)$ for representing the short rate process.

The fundamental data set is depicted in Figure 1. When this curve with fluctuations is viewed as the short rate process, it is evident that the process is not governed by a single set of the three parameters $\{\phi, \alpha, \sigma\}$, with $\phi$ for the market fitting factor, $\alpha$ for the reversion factor and $\sigma$ for the volatility. Accordingly, it is necessary to specify the state space $\mathcal{J}=\{0,1,2\}$ of $J(t)$ precisely, where state 0 means the bad economic condition, state 1 indicates the normal economic condition, and state 2 describes the good economic condition. In this regard, the range of the fundamental data set is decomposed into 500 intervals of equal length. The corresponding cumulative distribution of the monthly data is illustrated in Figure 2. In order to define the state space $\mathcal{J}=\{0,1,2\}$, we choose the 20 percentile point and the 40 percentile point, as shown by the two dotted lines in Figure 2 , 
with the inverted points of 0.026428 and 0.042513 respectively along the horizontal axis. Accordingly, the state space $\mathcal{J}$ is defined as described below. For notational convenience, we set $t=0$ at the beginning of October 1989 and $T>0$ to be the end of December 2008 . The interval $[0, T]$ is decomposed into monthly segments and those months are numbered consecutively. Let $m(t)$ be the month ID of time $t$.

$$
J(t)= \begin{cases}0 & \begin{array}{l}
\text { if the value of the monthly LIBOR for } m(t) \\
\text { is less than or equal to } 0.026428
\end{array} \\
\text { if the value of the monthly LIBOR for } m(t) & \begin{array}{l}
\text { is grater than } 0.026428 \text { but less than or equal to } 0.042513 \\
2 \quad \begin{array}{l}
\text { if the value of the monthly LIBOR for } m(t) \\
\text { is grater than } 0.042513
\end{array}
\end{array} .\end{cases}
$$

The state change borders are also indicated in Figure 1 by the two dotted lines.

[Figure 1 about here.]

[Figure 2 about here.]

For estimating the values of the birth-death process hazard rates $\eta_{0}^{+}, \eta_{1}^{-}, \eta_{1}^{+}$and $\eta_{2}^{-}$, we employ the maximum likelihood method. More formally, let $D_{i j}(t)$ be the number of transitions of $J(t)$ from state $i$ to state $j$ in the time interval $[0, t]$, and define $U_{i}(t)$ to be the total time spent in state $i$ in $[0, t]$. Then the maximum likelihood estimator of the hazard rate $\eta_{i j}$ of $J(t)$ can be given by

$$
\widehat{\eta}_{i j}(t)=\frac{D_{i j}(t)}{U_{i}(t)}
$$

see, e.g. Bladt and Sørensen (2005). Table 2 shows the maximum likelihood estimators of the hazard rates of $J(t)$.

[Table 2 about here.]

As discussed in Section 3, given $J(t)=j$, the short rate process behaves like a Vasicek model with constant parameters $\phi_{j}, \alpha_{j}$ and $\sigma_{j}$ until $J(t)$ changes its state. The three parameters can be estimated with Generalized Method of Moments of Hansen (1982). The reader is referred to Chan et al. (1992) for further details. The data corresponding to state $j$ are used together in estimating $\phi_{j}, \alpha_{j}$ and $\sigma_{j}$. Tabel 3 exhibits the estimation results. For comparison, the estimators for the Vasicek model without the macro-economic condition are also shown, which can be obtained by using the whole data together. It is worth noting that the volatility for the Vasicek model without the macro-economic condition is 0.010749, which is much larger than the Vasicek model with the macroeconomic condition, as one may expect. From Equation (3.6), one sees that $\phi_{j} / \alpha_{j}$ is the value to which the short rate process approaches when the age of $J(\cdot)$ at state $j$ becomes longer. Consequently, it is natural to assume that this value increases as the macro-economic condition becomes better, as can be observed in Tabel 3.

[Table 3 about here.]

We are now in a position to test the speed and efficiency of Algorithms A.2 through A.5. The following European option defined on a zero-coupon discount bond is considered as a base for this purpose. 


\section{Example of European Call Option Defined on a Zero-coupon Discount Bond}

- $\quad$ Maturity of bond: 2 years

- $\quad$ Face value of bond: $\$ 1$

- Initial value of short rate: 0.035

- Maturity of option: 1 year

- $\quad$ Strike price of option: $\$ 0.9$

- Length of unit time interval $\Delta: 1 / 100$ years

- $\quad$ Factor to define the number of states of Ehrenfest approximation $V: 50$

Table 4 provides the price of the above European call option, where the price is compared with the analytic solution of the Vasicek model without the macro-economic condition based on Hull and White (1990b). The former price is 0.07182 , which is higher than the latter price of 0.06604 . This may be so because the fundamental data set stayed in the good economic condition for a substantial amount of time, which would be captured less in the Vasicek model without the macro-economic condition than in our model with the macro-economic condition.

[Table 4 about here.]

Exhibited in Figure 3 are the tradeoffs between the computational time and the two accuracy factors: the precision of the discrete approximation of the short rate represented by $V$ and the precision of the discrete approximation of the time axis captured by $\Delta$. One sees that the computational time increases as $V$ increases and $\Delta$ decreases, i.e., the discrete approximations approach the continuum. However, this increase of the computational time as a function of $\Delta$ is fairly contained and is almost linear for $V=30$ or less, with the maximum computational time of 8.7 seconds at $V=30$ and $\Delta=1 / 100$. Considering the fact that the convergence accuracy at $V=30$ and $\Delta=1 / 100$ in comparison with the computed price at $V=50$ and $\Delta=1 / 100$ can be given as $0.05 \%$ in the relative error, the proposed algorithms provide the speed and efficiency that would tolerate the repeated computations. We also note that the computed option price decreases as $V$ increases and $\Delta$ decreases.

In Tables 5 and 6 , the sensitivities of the computed option price are illustrated as the initial short rate and the strike price vary respectively. The corresponding elasticities are defined as

$$
\begin{aligned}
& \text { Elasticity for Tabel } 5=\frac{\operatorname{DIFF}(\text { Price }) / \text { Price }}{\operatorname{DIFF}(\text { Initial Short Rate }) / \text { Initial Short Rate }}, \\
& \text { Elasticity for Tabel } 6=\frac{\operatorname{DIFF}(\text { Price }) / \text { Price }}{\operatorname{DIFF}(\text { Strike Price }) / \text { Strike Price }},
\end{aligned}
$$

where $\operatorname{DIFF}(\cdot)$ describes the difference of the corresponding values in two adjacent columns. We note that the price of the European call option decreases as either the initial short rate or the strike price increases, as expected. The price, however, is not so sensitive to 
the changes of the initial short rate, while the elasticity with respect to the price and the strike price is quite high, reaching $-1,211.53 \%$ when the strike price is changed from $\$ 0.90$ to $\$ 0.95$.

All the computations are done using MATLAB R2009b on an iMac with Mac OS X 1.6 Snow Leopard, 2.66GHz Intel Core 2 Duo processor and 4GB memory.

[Figure 3 about here.]

[Table 5 about here.]

[Table 6 about here.]

\section{Acknowledgement}

The authors with to thank Mr. Takamasa Kondo for helpful discussions.

\section{References}

M. Bladt and M. Sørensen. Statistical inference for discretely observed Markov jump processess. Journal of the Royal Statistical Society: Series B (Statistical Methodology), 67(3):395-410, 2005.

K. C. Chan, G. A. Karolyi, F. A. Longstaff, and A. B. Sanders. An Empirical Comparison of Alternative Models of the Short-Term Interest Rate. The Journal of Finance, XLVII (3):1209-1227, July 1992.

J. Gotoh, H. Jin, and U. Sumita. Numerical Evaluation of Dynamic Behavior of OrnsteinUhlenbeck Processes Modified by Various Boundaries and its Application to Pricing Barrier Options. Methodology and Computing in Applied Probability, 2009. DOI 10.1007/s11009-009-9152-4, Published online: 08 August 2009.

L. P. Hansen. Large Sample Properties of Generalized Method of Moments Estimators. Econometrica, 50(4):1029-1054, July 1982.

J. Hull and A. White. Valuing Derivative Securities Using the Explicit Finite Differential Method. Journal of Financial and Quantitative Analysis, 25(1):87-100, Mar 1990a.

J. Hull and A. White. Pricing Interest-Rate-Derivative Securities. The Review of Financial Studies, 3(4):573-592, 1990b.

H. Jin, J. Gotoh, and U. Sumita. A New Approach for Computing Option Prices of the Hull-White Type with Stepwise Reversion and Volatility Functions. The Journal of Derivatives, pages 67-85, Fall 2007.

J. Keilson. Markov Chain Models: Rarity and Exponentiality. Applied Mathematical Science Series (28). Springer-Verlag, 1979.

A. Pelsser. Efficient Methods for valuing Interest Rate Derivatives. Springer-Verlag London Limited, 2000. 
U. Sumita, J. Gotoh, and H. Jin. Numerical Exploration of Dynamic Behavior of OrnsteinUhlenbeck Processes via Ehrenfest Process Approximation. Journal of the Operations Research Society of Japan, 49(3):256-278, 2006.

O. Vasicek. An Equilibrium Characterization of the Term Structure. Journal of Financial Economics, 5:177-188, 1977. 


\section{Appendices}

\section{A Algorithms}

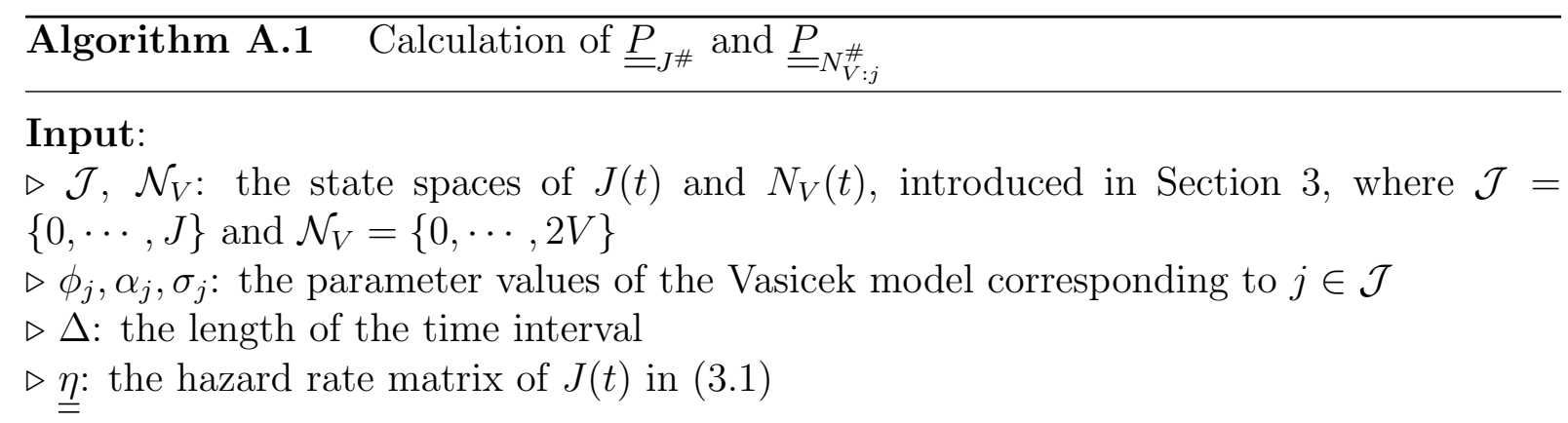

\section{Output:}

$\triangleright \underline{\underline{P}}_{J \#}$ : the one step transition probability matrix of $J^{\#}(\cdot)$

$\triangleright \underline{\underline{P}}_{N_{V: j}^{\#}}^{\#}$ : the one step transition probability matrix of $N_{V: j}^{\#}(\cdot)$ for all $j \in \mathcal{J}$

\section{Procedure:}

1: Compute $\underline{\underline{P}}_{J \#}$ and $\underline{\underline{P}}_{N_{V: j}^{\#}}$ for $j \in \mathcal{J}$ based on (4.4) and (4.9) respectively.

In what follows, the output matrices of Algorithm A.1 are assumed to be available. 


\footnotetext{
Algorithm A.2 Calculation of $\pi(j, \ell, n, k)$ and $\widehat{\chi}(j, \ell, n, k)$

\section{Input:}

$\triangleright k$ : the $k$-th discrete point in time

$\triangleright \mathcal{J} \times \mathbb{Z}_{+} \times \mathcal{N}_{V}$ : the state space of the trivariate process $\left[J^{\#}(k), Y^{\#}(k), N_{V: J \#(k)}^{\#}\left(Y^{\#}(k)\right)\right]$, introduced in Section 4 , where $\mathcal{J}=\{0, \cdots, J\}, \mathbb{Z}_{+}=\{0,1,2, \cdots\}$ and $\mathcal{N}_{V}=\{0, \cdots, 2 V\}$

$\triangleright[j, \ell, n] \in \mathcal{J} \times \mathbb{Z}_{+} \times \mathcal{N}_{V}$ : the current state of $\left[J^{\#}(k), Y^{\#}(k), N_{V: J \#(k)}^{\#}\left(Y^{\#}(k)\right)\right]$

$\triangleright \phi_{j}, \alpha_{j}, \sigma_{j}$ : the parameter values of the Vasicek model corresponding to $j \in \mathcal{J}$

$\triangleright \Delta$ : the length of the time interval

$\triangleright \pi(j, 0, n, 0)$ : the initial state probability associated with state $[j, 0, n]$

$\triangleright \widehat{\chi}(j, 0, n, 0)$ : the initial value of the short rate associated with state $[j, 0, n]$
}

\section{Output:}

$\triangleleft \pi(j, \ell, n, k)$ : state probability at state $[j, \ell, n]$ at time $k \Delta$

$\triangleleft \widehat{\chi}(j, \ell, n, k)$ : value of short rate at state $[j, \ell, n]$ at time $k \Delta$

\section{Procedure:}

1: if $k=0$ then

2: $\quad \pi(j, \ell, n, k) \leftarrow \pi(j, 0, n, 0), \quad \widehat{\chi}(j, \ell, n, k) \leftarrow \widehat{\chi}(j, 0, n, 0)$.

3: else

4: $\quad$ if $\ell \neq 0$ then

5: $\quad$ Calculate $\pi(j, \ell, n, k)$ by using (4.11) and Algorithm A.2 recursively.

6: $\quad$ Calculate $\widehat{\chi}(j, \ell, n, k)$ by using (4.13) and Algorithm A.2 recursively.

7: else

8: $\quad$ Calculate $\pi(j, \ell, n, k)$ by using (4.12) and Algorithm A.2 recursively.

9: $\quad$ Calculate $\widehat{\chi}(j, \ell, n, k)$ by using (4.14) and Algorithm A.2 recursively.

10: end if

11: end if 


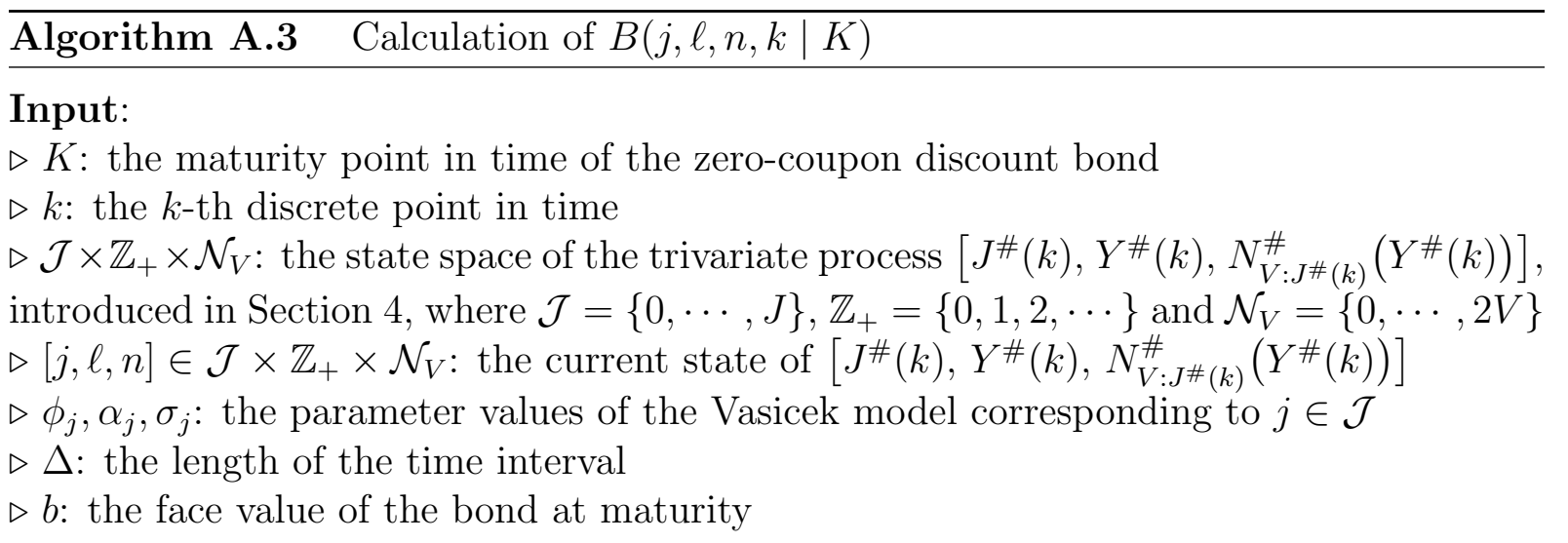

\section{Output:}

$\triangleleft B(j, \ell, n, k \mid K)$ : price of the zero-coupon discount bond at time $k \Delta$ with maturity $K$, where the corresponding trivariate process is at state $[j, \ell, n]$

\section{Procedure:}

1: if $k=K$ then

2: $\quad B(j, \ell, n, k \mid K) \leftarrow b$.

3: else

4: Calculate $B(j, \ell, n, k \mid K)$ by using (4.16), Algorithm A.2 and A.3 recursively.

5: end if

\section{Algorithm A.4 Calculation of $Q(j, \ell, n, k)$}

\section{Input:}

$\triangleright k$ : the $k$-th discrete point in time

$\triangleright \mathcal{J} \times \mathbb{Z}_{+} \times \mathcal{N}_{V}$ : the state space of the trivariate process $\left[J^{\#}(k), Y^{\#}(k), N_{V: J \#(k)}^{\#}\left(Y^{\#}(k)\right)\right]$, introduced in Section 4 , where $\mathcal{J}=\{0, \cdots, J\}, \mathbb{Z}_{+}=\{0,1,2, \cdots\}$ and $\mathcal{N}_{V}=\{0, \cdots, 2 V\}$ $\triangleright[j, \ell, n] \in \mathcal{J} \times \mathbb{Z}_{+} \times \mathcal{N}_{V}$ : the current state of $\left[J^{\#}(k), Y^{\#}(k), N_{V: J^{\#}(k)}^{\#}\left(Y^{\#}(k)\right)\right]$

$\triangleright \Delta$ : the length of the time interval

\section{Output:}

$\triangleleft Q(j, \ell, n, k)$ : present value of the Arrow-Debreu security with maturity price 1 at time $k \Delta$ given state $[j, \ell, n]$

\section{Procedure:}

1: if $k=0$ then

2: $\quad Q(j, \ell, n, k)=Q(j, 0, n, 0) \leftarrow \delta_{n V}$.

3: else

if $\ell=0$ then

5: $\quad$ Calculate $Q(j, 0, n, k)$ by using (4.18), Algorithm A.2 and A.4 recursively.

6: else

7: $\quad$ Calculate $Q(j, \ell, n, k)$ by using (4.19), Algorithm A.2 and A.4 recursively.

8: $\quad$ end if

9: end if 


\section{Algorithm A.5 $\quad$ Calculation of $\Pi_{C}(M, S \mid K)$ \\ Input: \\ $\triangleright K$ : the maturity point in time of the zero-coupon discount bond \\ $\triangleright M$ : the maturity point in time of the European call option defined on the discount bond \\ $\triangleright S$ : the strike price at maturity of the European call option}

\section{Output:}

$\triangleleft \Pi_{C}(M, S \mid K)$ : price of the European call option

\section{Procedure:}

1: $\Pi_{C}(M, S \mid K) \leftarrow 0$.

2: for $j \in \mathcal{J}$ do

3: $\quad$ for $\ell=0$ to $M$ do

4: $\quad$ for $n \in \mathcal{N}_{V}$ do

5: $\quad \Pi_{C}(M, S \mid K) \leftarrow \Pi_{C}(M, S \mid K)+[B(j, \ell, n, M \mid K)-S]^{+} Q(j, \ell, n, M)$,

where $B(j, \ell, n, M \mid K)$ and $Q(j, \ell, n, M)$ can be calculated by using Algorithm A.3 and A.4 respectively.

6: $\quad$ end for

7: $\quad$ end for

8: end for 


\section{List of Figures}

1 Monthly USD LIBOR and the States of Macro-economy. (The border line of the economy states are 0.026428 and 0.042513 .) . . . . . . . . . . . 20

2 Cumulative Distribution of Monthly USD LIBOR with Data Period from Sep. 1989 to Dec. 2008. . . . . . . . . . . . . . . . . . . . . 21

3 European Call Option Prices and Computational Times . . . . . . . . . . . 22 


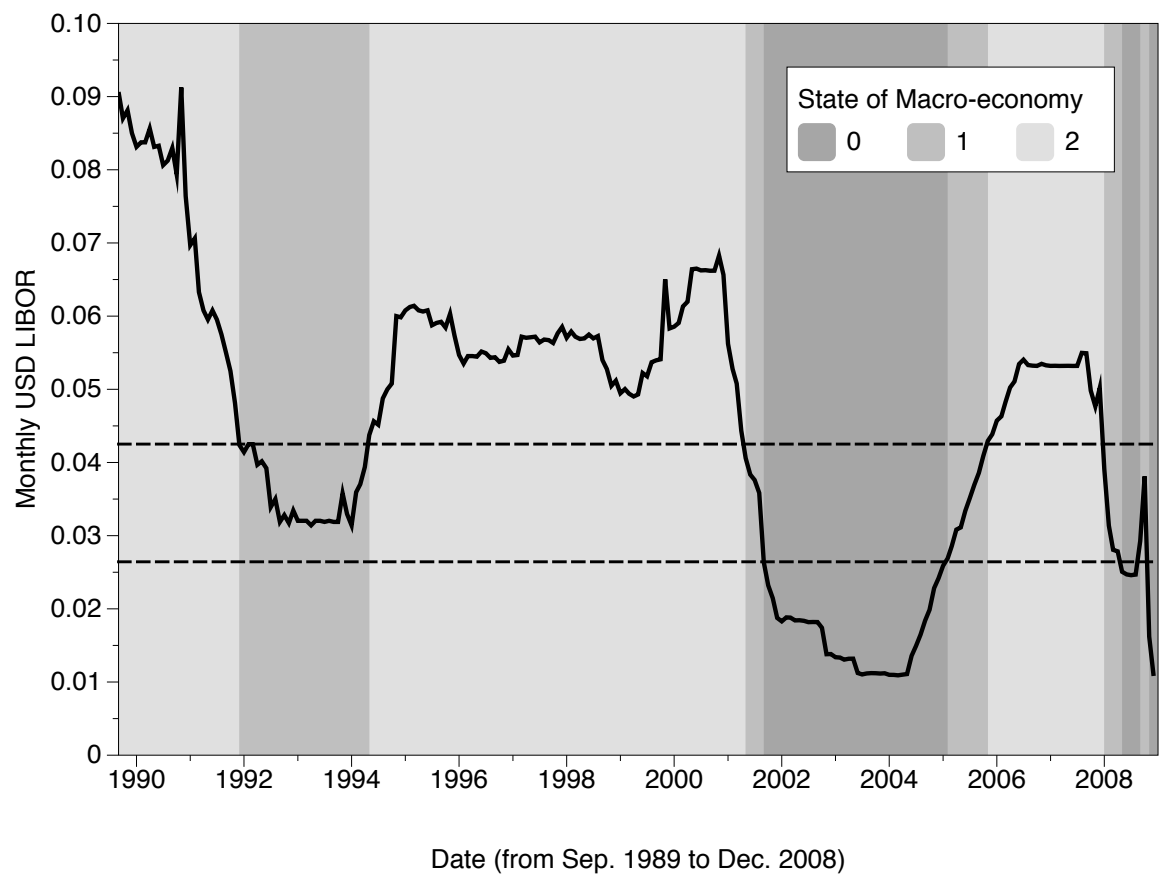

Figure 1: Monthly USD LIBOR and the States of Macro-economy. (The border line of the economy states are 0.026428 and 0.042513 .) 


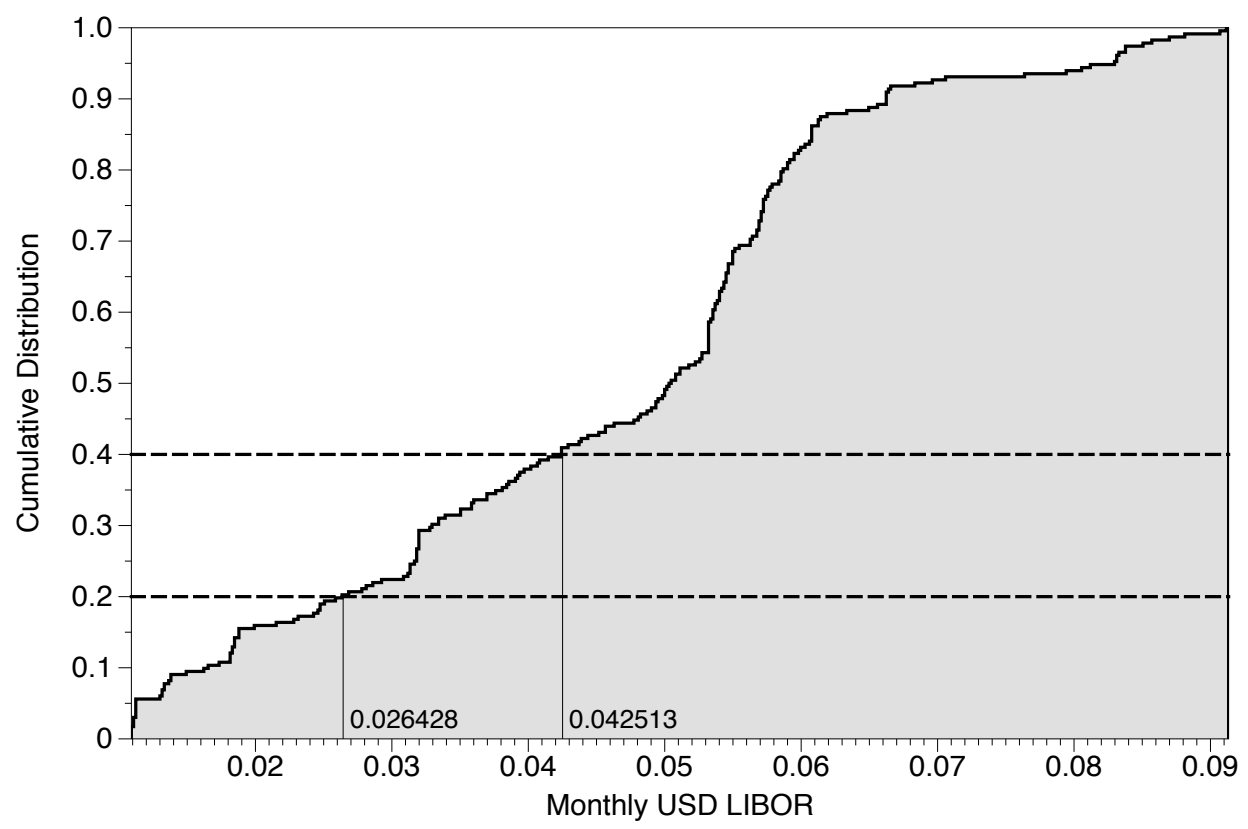

Figure 2: Cumulative Distribution of Monthly USD LIBOR with Data Period from Sep. 1989 to Dec. 2008. 


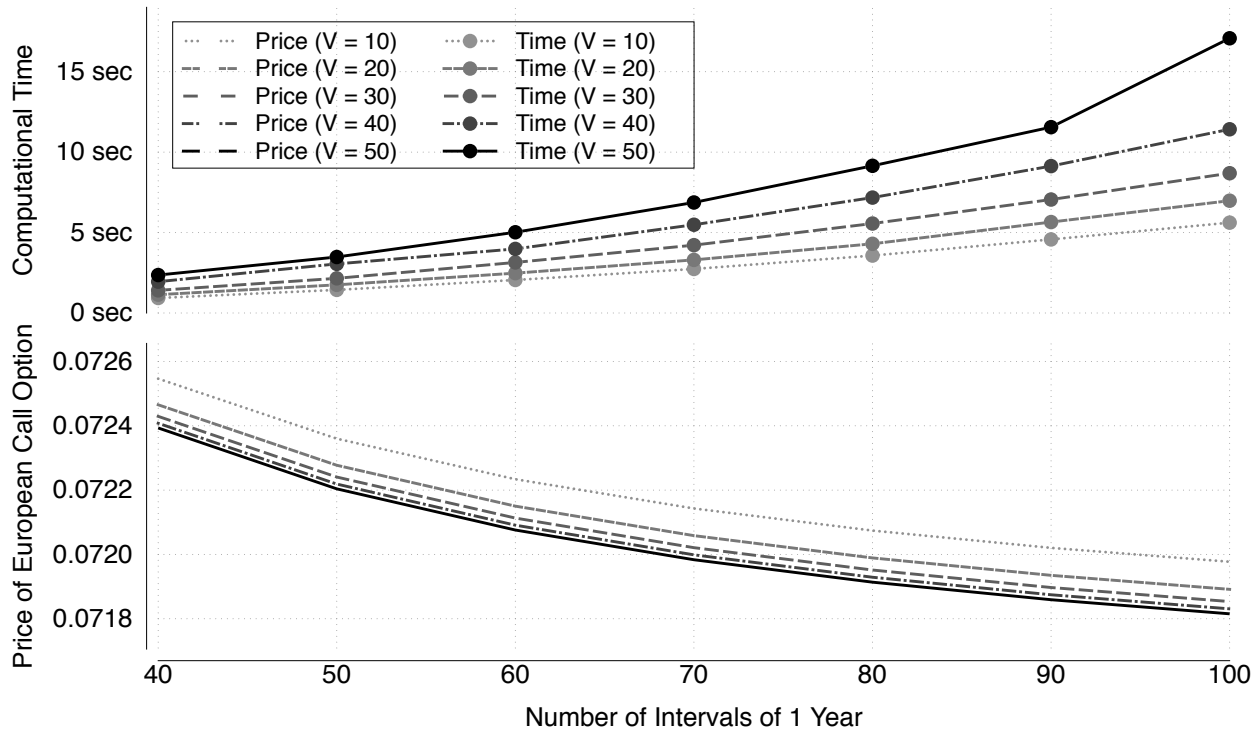

Figure 3: European Call Option Prices and Computational Times 


\section{List of Tables}

1 State Conversions . . . . . . . . . . . . . . . . . 24

2 Maximum Likelihood Estimators of Hazard Rates of $J(t) \ldots \ldots \ldots$

3 Estimated Parameters of Vasicek Models . . . . . . . . . . . . . 26

4 Prices of European Bond Call Option . . . . . . . . . . . . . . . . 27

5 European Bond Call Option Prices with Various Initial Short Rates . . . . 28

6 European Bond Call Option Prices with Various Strike Prices . . . . . . . 29 
Table 1: State Conversions

\begin{tabular}{|c|c|c|c|}
\hline \multirow{2}{*}{ Process } & \multicolumn{2}{|c|}{ State Conversion } & State Space \\
\cline { 2 - 3 } & $x \in \mathbb{R} \rightarrow n \in \mathcal{N}_{V}$ & $n \in \mathcal{N}_{V} \rightarrow x \in \mathbb{R}$ & \\
\hline \hline$N_{V}(t)$ & $n=\xi_{V}(x)+V$ & $n$ & $\mathcal{N}_{V}=\{0,1, \cdots, 2 V\}$ \\
\hline$X_{V}(t)$ & $\frac{\sigma}{\sqrt{\alpha V}} \xi_{V}(x)$ & $x_{V}(n)=\frac{\sigma}{\sqrt{\alpha V}} n-\sigma \sqrt{\frac{V}{\alpha}}$ & $\mathcal{X}_{V}=\left\{-\sigma \sqrt{\frac{V}{\alpha}}, \cdots, \sigma \sqrt{\frac{V}{\alpha}}\right\}$ \\
\hline$X_{O U}(t)$ & $x$ & $x=x_{V}(n)$ & $\mathbb{R}=\{-\infty,+\infty\}$ \\
\hline
\end{tabular}


Table 2: Maximum Likelihood Estimators of Hazard Rates of $J(t)$

\begin{tabular}{cccc}
\hline$\eta_{0}^{+}$ & $\eta_{1}^{-}$ & $\eta_{1}^{+}$ & $\eta_{2}^{-}$ \\
\hline 0.5106 & 0.7500 & 0.5000 & 0.2627 \\
\hline
\end{tabular}


Table 3: Estimated Parameters of Vasicek Models

\begin{tabular}{ccccc}
\hline Economy State $j$ & $\phi_{j}$ & $\alpha_{j}$ & $\sigma_{j}$ & $\phi_{j} / \alpha_{j}$ \\
\hline 0 & 0.017998 & 1.253164 & 0.002918 & 0.014362 \\
\hline 1 & 0.104914 & 3.039290 & 0.009231 & 0.034519 \\
\hline 2 & 0.039756 & 0.721681 & 0.007961 & 0.055579 \\
\hline \hline
\end{tabular}

Vasicek Model without

the Macro-economic Condition $\quad 0.003766 \quad 0.166188 \quad 0.010749 \quad 0.022659$ 
Table 4: Prices of European Bond Call Option

\begin{tabular}{cc}
\hline Proposed Approach & $\begin{array}{c}\text { Analytic Solution without the } \\
\text { Macro-economic Condition }\end{array}$ \\
\hline 0.07182 & 0.06604 \\
\hline
\end{tabular}

Note: initial short rate $=0.035$, face value of bond $=\$ 1$, bond maturity $=2$ years, option maturity $=1$ year, strike price $=\$ 0.9$, length of unit time interval $\Delta=1 / 100$ years, $V=50$. 
Table 5: European Bond Call Option Prices with Various Initial Short Rates

\begin{tabular}{cccccccc}
\hline & \multicolumn{7}{c}{ Initial Short Rate } \\
\cline { 2 - 8 } & 0.020 & 0.025 & 0.030 & 0.035 & 0.040 & 0.045 & 0.050 \\
\hline Price & 0.07360 & 0.07300 & 0.07241 & 0.07182 & 0.07123 & 0.07064 & 0.07006 \\
\hline Elasticity & & $-3.26 \%$ & $-4.04 \%$ & $-4.89 \%$ & $-5.75 \%$ & $-6.63 \%$ & $-7.39 \%$ \\
\hline
\end{tabular}

Note: face value of bond $=\$ 1$, bond maturity $=2$ years, option maturity $=1$ year, strike price $=\$ 0.9$, length of unit time interval $\Delta=1 / 100$ years, $V=50$. 
Table 6: European Bond Call Option Prices with Various Strike Prices

\begin{tabular}{cccccc}
\hline & \multicolumn{5}{c}{ Strike Price $(\$)$} \\
\cline { 2 - 6 } & 0.75 & 0.80 & 0.85 & 0.90 & 0.95 \\
\hline Price & 0.21684 & 0.16850 & 0.12016 & 0.07182 & 0.02348 \\
\hline Elasticity & & $-334.39 \%$ & $-459.01 \%$ & $-683.90 \%$ & $-1,211.53 \%$ \\
\hline
\end{tabular}

Note: initial short rate $=0.035$, face value of bond $=\$ 1$, bond maturity $=2$ years, option maturity $=1$ year, length of unit time interval $\Delta=1 / 100$ years, $V=50$. 